\title{
Cutaneous vs. Mucosal Tropism: The Papillomavirus Paradigm Comes to an "and"
}

\author{
Gennaro Altamura ${ }^{1}$, Massimo Tommasino ${ }^{2}$ and Giuseppe Borzacchiello ${ }^{1 *}$ \\ ${ }^{1}$ Department of Veterinary Medicine and Animal Productions, University of Naples Federico II, Naples, Italy, ${ }^{2}$ nfections and \\ Cancer Biology Group, International Agency for Research on Cancer (IARC), Lyon, France
}

Keywords: papillomavirus, tropism, epithelia, mucosa, skin, HPV, FcaPV, EcPV

\section{INTRODUCTION}

Papillomaviruses (PVs) are small, double stranded DNA viruses with a preferential tropism for epithelial tissues (Van Doorslaer et al., 2018). Approximately 300 PV types have been isolated so far and placed in the taxonomy, however several studies indicate that many more PV types exist (Araldi et al., 2017; Brancaccio et al., 2018; Galati et al., 2020). The canonical criteria for the placement of new isolates in the phylogenetic tree, which are based on the percentage of similarity with other known PVs within the L1 gene sequence, establish that a PV is classified as a new viral type when the difference is $>10 \%$ (de Villiers, 2013). All PV types belong to the family of Papillomaviridae which, according to the most recent classification, is split in two subfamilies sharing $<45 \%$ of sequence identity in L1, namely Firstpapillomavirinae and Secondpapillomavirinae (Van Doorslaer et al., 2018). Subfamilies are divided in subgroups called genera ( $\alpha, \beta, \gamma$, and so on), which are further subdivided into species (numbered as 1, 2, 3, and so on) (de Villiers, 2013). Each of the genera can include animal and human PVs (HPVs), which are numbered consecutively in order of discovery. Secondpapillomavirinae currently includes a single genus (Alefpapillomavirus) and a unique viral species, isolated from a skin lesion of a fish (gilt-head sea bream, Sparus aurata) (Van Doorslaer et al., 2018).

Beyond the conventional criteria for classification according to L1 sequence, non-canonical ways based on biological properties have become of common use. For instance, mucosal $\alpha$-HPVs comprise high-risk (HR) or low-risk types, according to their role in causing malignant or benign lesions, respectively (Zur Hausen, 2002).

In addition, PVs can be classified as cutaneous or mucosal, based on their ability to infect cutaneous or mucosal epithelia (de Villiers, 2013). Over decades of research, this tropism-based nomenclature has become almost a paradigm, which has led to PVs affinity for skin or mucosal epithelia being considered as mutually exclusive (de Villiers, 2013).

However, a series of recent studies indicate that specific subgroups of animal and human PVs could retain a dual tropism.

a section of the journal

Frontiers in Microbiology

Received: 05 August 2020

Accepted: 14 September 2020

Published: 14 October 2020

Citation:

Altamura G, Tommasino M and

\section{BREAKING THE PARADIGM: RECENT EVIDENCE ABOUT ANIMAL AND HUMAN PVs}

Studies of feline PVs indicate that the same virus can be associated with development of cancers of the skin and mucosal epithelia. Felis catus PV type 2 (FcaPV-2), originally isolated from and then widely associated with pre-neoplastic and neoplastic skin lesions, is detectable in a subset of feline oral squamous cell carcinoma (FOSCC) samples and FOSCC-derived cell lines, where it is transcriptionally active (Lange et al., 2009; Altamura et al., 2018a, 2020; Munday et al., 2018). Accordingly, molecular studies clearly show that FcaPV-2 E6 and E7 oncogenes display 
biological properties similar to those of mucosal HR HPV-16 in degrading p53 and pRb tumor suppressor proteins (Altamura et al., 2016, 2018a). Possible transmission routes between the two anatomical sites have also been hypothesized, such as via skin licking or through the bloodstream carrying viral particles to the epithelium of oral cavity (Altamura et al., 2018b, 2020). Of note, other FcaPVs are suspected to infect skin and oral epithelia, such as FcaPV-1, -3, and -4 (Munday and French, 2015; Munday et al., 2015, 2018; Mazzei et al., 2018; Vascellari et al., 2019; Chu et al., 2020), however additional experimental data are warranted for any confirmation.

New evidence also comes from Equus caballus PV type 2 (EcPV-2). It is recognized as the causative agent of neoplastic lesions arising on the skin of external genitalia of the horses; indeed, EcPV-2 DNA and mRNA are consistently found in equine penile SCC and relative precursors (Sykora and Brandt, 2017). More precisely, EcPV-2 induced diseases develop preferentially at mucocutaneous junctions, suggesting a possible affinity for cells of both the skin and mucosal epithelia of genital region (Sykora and Brandt, 2017; Ramsauer et al., 2019). Interestingly, the presence of EcPV-2 has also been reported in a subset of equine head and neck cancers (HNCs), and recent work shows that EcPV-2 is detectable in neoplastic cells of equine gastric SCC (Knight et al., 2013; Sykora et al., 2017; Alloway et al., 2020).

It is also worth mentioning the growing body of evidence for expanded tissue tropism exhibited by the recently discovered Mus musculus PV (MmuPV-1). Similarly to HR-HPVs, MmuPV1 is sexually transmitted, however it can spread from ano-genital mucosa to different cutaneous sites and vice versa and cause development of neoplastic lesions in all of these anatomical regions (Cladel et al., 2017; Spurgeon and Lambert, 2019). Interestingly, MmuPV-1 E6 and E7 retain several molecular activities associated with cell transformation by mucosal HR-HPVs, despite the limited similarity in the nucleotide sequence of their respective oncogenes (Spurgeon and Lambert, 2020).

Studies of HPVs confirm that some viral types have a dual tropism. HPVs of genus $\beta$ are classified as cutaneous by assumption, given that most, if not all, of them have been isolated from the skin (Quint et al., 2015). $\beta-1$ and $\beta-2 \mathrm{HPV}$ types are suspected to be major players in the development of cutaneous SCC in cooperation with UV exposure, particularly in patients with immunosuppressive disorders and transplants recipients (Bandolin et al., 2020). However, a series of reports show that DNA from $\beta$-HPVs can be consistently found in other anatomical sites in addition to skin, such as external genital skin, nasal cavity, anal and oral mucosa, where their presence is even associated with increased risk of HNC (Bottalico et al., 2011; Pierce Campbell et al., 2013; Torres et al., 2015; Agalliu et al., 2016; Donà et al., 2016; Nunes et al., 2016). Prevalence and concordance studies at cutaneous and mucosal sites also suggest a sexual transmission route, and that fingernails may be a source of autoinoculation of $\beta$-HPVs from skin to the oral cavity (Hampras et al., 2017; Winer et al., 2019). In this context, particular mention should be made of HPV types belonging to the species $\beta-3$, especially HPV-49, -75 , and -76 . They display transforming properties in primary human keratinocytes comparable to those of HPV-16 (Cornet et al., 2012; Minoni et al., 2020). Most importantly, some $\beta-3-\mathrm{HPV}$ types retain the ability to induce p53 degradation with a similar mechanism to HPV-16 (Cornet et al., 2012; Minoni et al., 2020). Moreover, HPV-49 E6 and E7 transgenic mice models show higher susceptibility to upper digestive tract cancers, with a molecular signature mimicking the damage induced by tobacco exposure (Viarisio et al., 2016, 2019).

\section{DISCUSSION}

In light of these recent lines of evidence, we believe that the conventional criteria for tropism classification need to be reconsidered. The assignment of PV types to genus and species of the phylogenetic tree is based merely on the nucleotide sequence of L1 gene and should not be assumed to reflect biological features nor to provide any definitive tropism information (de Villiers, 2013; Minoni et al., 2020). Recent epidemiological and experimental data on $\beta$-HPVs are in line with this hypothesis, and this is evident also for genus $\alpha$, which groups both cutaneous and mucosal HPVs (Minoni et al., 2020). Therefore, the characterization of biological properties, beyond the comparative analysis of genetic sequences, appears to be necessary for a comprehensive evaluation of viral tropism, as shown also by studies of animal PVs (Altamura et al., 2016, 2018a; Alloway et al., 2020; Minoni et al., 2020). Moreover, the search for each human and animal PV should be expanded across a wide spectrum of anatomical sites, regardless of which body region they were initially isolated from, because limiting to one site may lead to an underestimation of their oncogenic potential at additional locations, even those coated by different types of epithelium.

Some evolutionary considerations should encourage colleagues to welcome our dissertation. It is well-known that completion of PVs life cycle with production of viral particles is strictly dependent on terminal differentiation of keratinocytes, thus productive infections of mucosal epithelia, which undergo incomplete keratinization, release few amounts of mature virions compared to those of the skin (Cubie, 2013). However, this in turn provides an advantage in terms of evasion of immune surveillance and viral persistence, therefore it would plausible that some cutaneous PVs evolved to exert also mucosal tropism in an attempt to establish a trade-off between efficient virus production and longer persistent infection. The recent development of MmuPV-1 infection models offers a significant opportunity to unravel the molecular mechanisms underlying these peculiar tropism features and other aspects of PVs biology impossible to study in classical transgenic mice (Spurgeon and Lambert, 2020).

We are confident that these considerations will be taken into account by scientists dealing with the issue of PVs tropism in the near future. 
In conclusion, it is reasonable to foreshadow that the paradigm is going to fall. Therefore, researchers in the field should no longer just establish whether a PV is cutaneous or mucosal; rather, they should consider the possibility of it being cutaneous and mucosal.

\section{AUTHOR CONTRIBUTIONS}

GA, MT, and GB conceived the study and drafted the manuscript. All authors contributed to the article and approved the submitted version.

\section{REFERENCES}

Agalliu, I., Gapstur, S., Chen, Z., Wang, T., Anderson, R. L., Teras, L., et al. (2016). Associations of oral alpha-, beta-, and gamma-human papillomavirus types with risk of incident head and neck cancer. JAMA Oncol. 2, 599-606. doi: 10.1001/jamaoncol.2015.5504

Alloway, E., Linder, K., May, S., Rose, T., DeLay, J., Bender, S., et al. (2020). A subset of equine gastric squamous cell carcinomas is associated with equus caballus papillomavirus-2 infection. Vet. Pathol. 57, 427-431. doi: 10.1177/0300985820908797

Altamura, G., Cardeti, G., Cersini, A., Eleni, C., Cocumelli, C., Bartolomé Del Pino, L. E., et al. (2020). Detection of Felis catus papillomavirus type-2 DNA and viral gene expression suggest active infection in feline oral squamous cell carcinoma. Vet. Comp. Oncol. 2020, 1-8. doi: 10.1111/vco.12569

Altamura, G., Corteggio, A., Pacini, L., Conte, A., Pierantoni, G. M., Tommasino, M., et al. (2016). Transforming properties of Felis catus papillomavirus type 2 E6 and E7 putative oncogenes in vitro and their transcriptional activity in feline squamous cell carcinoma in vivo. Virology 496, 1-8. doi: 10.1016/j.virol.2016.05.017

Altamura, G., Jebara, J., Cardeti, G., and Borzacchiello, G. (2018b). Felis catus papillomavirus type-2 but not type-1 is detectable and transcriptionally active in the blood of healthy cats. Transbound. Emerg. Dis. 65, 497-503. doi: $10.1111 /$ tbed.12732

Altamura, G., Power, K., Martano, M., Degli Uberti, B., Galiero, G., De Luca, G., et al. (2018a). Felis catus papillomavirus type-2 E6 binds to E6AP, promotes E6AP/p53 binding and enhances p53 proteasomal degradation. Sci. Rep. 8:17529. doi: 10.1038/s41598-018-35723-7

Araldi, R. P., Assaf, S. M. R., Carvalho, R. F., Carvalho, M. A. C. R., Souza, J. M., Magnelli, R. F., et al. (2017). Papillomaviruses: a systematic review. Genet. Mol. Biol. 40, 1-21. doi: 10.1590/1678-4685-gmb-20 16-0128

Bandolin, L., Borsetto, D., Fussey, J., Da Mosto, M. C., Nicolai, P., Menegaldo, A., et al. (2020). Beta human papillomaviruses infection and skin carcinogenesis. Rev. Med. Virol. 30:e2104. doi: 10.1002/rmv.2104

Bottalico, D., Chen, Z., Dunne, A., Ostoloza, J., McKinney, S., Su, C., et al. (2011). The oral cavity contains abundant known and novel human papillomaviruses from the Betapapillomavirus and Gammapapillomavirus genera. J. Infect. Dis. 204, 787-792. doi: 10.1093/infdis/jir383

Brancaccio, R. N., Robitaille, A., Dutta, S., Cuenin, C., Santare, D., Skenders, G., et al. (2018). Generation of a novel next-generation sequencing-based method for the isolation of new human papillomavirus types. Virology. 520, 1-10. doi: 10.1016/j.virol.2018.04.017

Chu, S., Wylie, T. N., Wylie, K. M., Johnson, G. C., Skidmore, Z. L., Fleer, M., et al. (2020). A virome sequencing approach to feline oral squamous cell carcinoma to evaluate viral causative factors. Vet. Microbiol. 240:108491. doi: 10.1016/j.vetmic.2019.108491

Cladel, N. M., Budgeon, L. R., Cooper, T. K., Balogh, K. K., Christensen, N. D., Myers, R., et al. (2017). Mouse papillomavirus infections spread to cutaneous sites with progression to malignancy. J. Gen. Virol. 98, 2520-2529. doi: 10.1099/jgv.0.000926

Cornet, I., Bouvard, V., Campo, M. S., Thomas, M., Banks, L., Gissmann, L., et al. (2012). Comparative analysis of transforming properties of E6 and

\section{FUNDING}

This work was funded by University of Naples Federico II. This work was partly supported by Fondation ARC pour la recherche sur le cancer (no. PJA 20151203192 to MT) (https:// www.fondation-arc.org/espace-chercheur).

\section{ACKNOWLEDGMENTS}

The authors thank Dr. Karen Müller for editing the manuscript.

E7 from different beta human papillomavirus types. J. Virol. 86, 2366-2370. doi: 10.1128/JVI.06579-11

Cubie, H. A. (2013). Diseases associated with human papillomavirus infection. Virology 445, 21-34. doi: 10.1016/j.virol.2013.06.007

de Villiers, E. M. (2013). Cross-roads in the classification of papillomaviruses. Virology 445, 2-10. doi: 10.1016/j.virol.2013.04.023

Donà, M. G., Gheit, T., Vescio, M. F., Latini, A., Moretto, D., Benevolo, M., et al. (2016). Incidence, clearance and duration of cutaneous beta and gamma human papillomavirus anal infection. J. Infect. 73, 380-383. doi: 10.1016/j.jinf.2016.07.006

Galati, L., Brancaccio, R. N., Robitaille, A., Cuenin, C., Luzi, F., Fiorucci, G., et al. (2020). Detection of human papillomaviruses in paired healthy skin and actinic keratosis by next generation sequencing. Papillomavirus Res. 9:100196. doi: 10.1016/j.pvr.2020.100196

Hampras, S. S., Rollison, D. E., Giuliano, A. R., McKay-Chopin, S., Minoni, L., Sereday, K., et al. (2017). Prevalence and concordance of cutaneous beta human papillomavirus infection at mucosal and cutaneous sites. J. Infect. Dis. 216, 92-96. doi: 10.1093/infdis/jix245

Knight, C. G., Dunowska, M., Munday, J. S., Peters-Kennedy, J., and Rosa, B. V. (2013). Comparison of the levels of Equus caballus papillomavirus type 2 (EcPV-2) DNA in equine squamous cell carcinomas and noncancerous tissues using quantitative PCR. Vet. Microbiol. 166, 257-262. doi: 10.1016/j.vetmic.2013.06.004

Lange, C. E., Tobler, K., Markau, T., Alhaidari, Z., Bornand, V., Stockli, R., et al. (2009). Sequence and classification of FdPV2, a papillomavirus isolated from feline Bowenoid in situ carcinomas. Vet. Microbiol. 137, 60-65. doi: 10.1016/j.vetmic.2009.01.002

Mazzei, M., Forzan, M., Carlucci, V., Anfossi, A. G., Alberti, A., Albanese, F., et al. (2018). A study of multiple Felis catus papillomavirus types $(1,2,3,4)$ in cat skin lesions in Italy by quantitative PCR. J. Feline Med. Surg. 20, 772-779. doi: 10.1177/1098612X17732255

Minoni, L., Romero-Medina, M., Venuti, A., Sirand, C., Robitaille, A., Altamura, G., et al. (2020). Transforming properties of beta-3 human papillomavirus E6 and E7 proteins. $m$ Sphere 5:e0398-20. doi: 10.1128/mSphere.00398-20

Munday, J. S., Fairley, R. A., Mills, H., Kiupel, M., and Vaatstra, B. L. (2015). Oral Papillomas associated with Felis catus papillomavirus type 1 in 2 domestic cats. Vet. Pathol. 52, 1187-1190. doi: 10.1177/0300985814565133

Munday, J. S., and French, A. F. (2015). Felis catus papillomavirus types 1 and 4 are rarely present in neoplastic and inflammatory oral lesions of cats. Res. Vet. Sci. 100, 220-222. doi: 10.1016/j.rvsc.2015.03.002

Munday, J. S., Sharp, C. R., and Beatty, J. A. (2018). Novel viruses: update on the significance of papillomavirus infections in cats. J. Feline Med. Surg. 21, 409-418. doi: 10.1177/1098612X18808105

Nunes, E. M., Sudenga, S. L., Gheit, T., Tommasino, M., Baggio, M. L., Ferreira, S., et al. (2016). HIM Study Group. Diversity of beta-papillomavirus at anogenital and oral anatomic sites of men: the HIM Study. Virology 495, 33-41. doi: 10.1016/j.virol.2016.04.031

Pierce Campbell, C. M., Messina, J. L., Stoler, M. H., Jukic, D. M., Tommasino, M., Gheit, T., et al. (2013). Cutaneous human papillomavirus types detected on the surface of male external genital lesions: a case series within the HPV infection in men study. J. Clin. Virol. 58, 652-659. doi: 10.1016/j.jcv.2013. 10.011 
Quint, K. D., Genders, R. E., de Koning, M. N. C., Borgogna, C., Gariglio, M., Bouwes Bavinck, J. N., et al. (2015). Human Beta-papillomavirus infection and keratinocyte carcinomas. J. Pathol. 235, 342-354. doi: 10.1002/path.4425

Ramsauer, A. S., Wachoski-Dark, G. L., Fraefel, C., Tobler, K., Brandt, S., Knight, C. G., et al. (2019). Paving the way for more precise diagnosis of EcPV2-associated equine penile lesions. BMC Vet. Res. 15:356. doi: 10.1186/s12917-019-2097-0

Spurgeon, M. E., and Lambert, P. F. (2019). Sexual transmission of murine papillomavirus (MmuPV1) in Mus musculus. eLife 8:e50056. doi: 10.7554/eLife.50056

Spurgeon, M. E., and Lambert, P. F. (2020). Mus musculus papillomavirus 1: a new frontier in animal models of papillomavirus pathogenesis. J. Virol. 94:e00002-20 doi: 10.1128/JVI.00002-20

Sykora, S., and Brandt, S. (2017). Papillomavirus infection and squamous cell carcinoma in horses. Vet. J. 223, 48-54. doi: 10.1016/j.tvjl.2017.05.007

Sykora, S., Jindra, C., Hofer, M., Steinborn, R., and Brandt, S. (2017). Equine papillomavirus type 2: an equine equivalent to human papillomavirus 16 ? Vet. J. 225, 3-8. doi: 10.1016/j.tvjl.2017.04.014

Torres, M., Gheit, T., McKay-Chopin, S., Rodríguez, C., Del Romero, J., Filotico, R., et al. (2015). Prevalence of beta and gamma human papillomaviruses in the anal canal of men who have sex with men is influenced by HIV status. J. Clin. Virol. 67, 47-51. doi: 10.1016/j.jcv.2015.04.005

Van Doorslaer, K., Chen, Z., Bernard, H. U., Chan, P. K. S., DeSalle, R., Dillner, J., et al. (2018) ICTV virus taxonomy profile: papillomaviridae. J. Gen. Virol. 99, 989-990. doi: 10.1099/jgv.0.0 01105

Vascellari, M., Mazzei, M., Zanardello, C., Melchiotti, E., Albanese, F., Forzan, M., et al. (2019). Felis catus papillomavirus types 1, 2, 3, 4, and 5 in feline Bowenoid in situ carcinoma: an in situ hybridization study. Vet. Pathol. 56, 818-825. doi: 10.1177/0300985819859874

Viarisio, D., Müller-Decker, K., Zanna, P., Kloz, U., Aengeneyndt, B., Accardi, R., et al. (2016). Novel B-HPV49 transgenic mouse model of upper digestive tract cancer. Cancer Res. 76, 4216-4225. doi: 10.1158/0008-5472.CAN-1 6-0370

Viarisio, D., Robitaille, A., Müller-Decker, K., Flechtenmacher, C., Gissmann, L., and Tommasino, M. (2019). Cancer susceptibility of beta HPV49 E6 and E7 transgenic mice to 4-nitroquinoline 1-oxide treatment correlates with mutational signatures of tobacco exposure. Virology 538, 53-60. doi: 10.1016/j.virol.2019.09.010

Winer, R. L., Gheit, T., Feng, Q., Stern, J. E., Lin, J., Cherne, S., et al. (2019). Prevalence and correlates of $\beta$ - and $\gamma$-human papillomavirus detection in oral samples from mid-adult women. J. Infect. Dis. 219, 1067-1075. doi: 10.1093/infdis/jiy632

Zur Hausen, H. (2002). Papillomaviruses and cancer: from basic studies to clinical application. Nat. Rev. Cancer. 2, 342-350. doi: 10.1038/nrc798

Disclaimer: Where the authors are identified as personnel of the International Agency for Research on Cancer/World Health Organization, the authors alone are responsible for the views expressed in this article and they do not necessarily represent the decisions, policy, or views of the International Agency for Research on Cancer/World Health Organization.

Conflict of Interest: The authors declare that the research was conducted in the absence of any commercial or financial relationships that could be construed as a potential conflict of interest.

Copyright (c) 2020 Altamura, Tommasino and Borzacchiello. This is an open-access article distributed under the terms of the Creative Commons Attribution License (CC $B Y)$. The use, distribution or reproduction in other forums is permitted, provided the original author(s) and the copyright owner(s) are credited and that the original publication in this journal is cited, in accordance with accepted academic practice. No use, distribution or reproduction is permitted which does not comply with these terms. 